\title{
The diameter of the circumcircle of a Heron triangle
}

\author{
Ronald van Luijk \\ Ronald van Luijk received his Ph.D. from the University of California at Berkeley in \\ 2005. Since then he has been affiliated as a postdoc with CRM (Montreal), MSRI \\ (Berkeley), PIMS/UBC/SFU (Vancouver), and as a visiting professor with Universi- \\ dad de los Andes, Bogotá, Colombia.
}

A Heron triangle is a triangle with integral sides and integral area. The circumradius and circumdiameter of a triangle are respectively the radius and the diameter of its circumcircle. In this article we will give a necessary and sufficient condition for a positive integer to be the circumradius or the circumdiameter of some Heron triangle. It is stated in the following theorem:

Theorem 1. Let $k$ be a positive integer. Then the following are equivalent:

(1) There is a Heron triangle with circumradius $k$.

(2) There is a Heron triangle with circumdiameter $k$.

(3) There is a prime divisor $p$ of $k$ satisfying $p \equiv 1 \bmod 4$.

This theorem generalizes the results of Kramer and Luca [2, Proposition 6] and Aassila [1, pp. 145-146]. They give identical proofs for the implication $(3) \Rightarrow(1)$. For the converse, Aassila had already conjectured that no prime $p$ satisfying $p \equiv 11 \bmod 12$ is the circumradius of a Heron triangle. This conjecture was proven by Kramer and Luca,

Ein Dreieck wird Heronsches Dreieck genannt, wenn seine Seitenlängen und sein Flächeninhalt ganzzahlig sind. Bekanntlich stellt die Heronsche Formel einen Zusammenhang zwischen den Seitenlängen und dem Flächeninhalt her. Verknüpft man auch noch den Umkreisdurchmesser mit diesen Grössen, so liegt die Frage nahe, welche ganzen Zahlen $k$ als Umkreisdurchmesser eines Heronschen Dreiecks vorkommen können. Es zeigt sich, dass darüber Teilbarkeitseigenschaften modulo 4 der Primteiler von $k$ entscheiden. Für den Beweis werden nur elementare Kenntnisse der Zahlentheorie und zwei elementargeometrische Formeln für den Flächeninhalt eines Dreiecks benutzt. Offen bleibt die Frage, welche rationalen Zahlen Umkreisdurchmesser eines Heronschen Dreiecks sein können. 
who showed that in fact no power of a prime $p$ with $p=2$ or $p \equiv 11 \bmod 12$ is the circumradius of a Heron triangle.

Question. The question of which rational numbers occur as the circumdiameter of a Heron triangle remains open. It follows from Theorem 1 that if $d$ is such a number, then there is a prime divisor $p$ of the numerator of $d$ that satisfies $p \equiv 1 \bmod 4$. We leave it as an exercise to the reader to show that every positive integer is the denominator of the circumdiameter of some Heron triangle.

To prove the implication (3) $\Rightarrow(1)$ of Theorem 1, papers [1] and [2] take any positive integer $k$ with a prime divisor $p$ as in (3). Both first show that there is a triangle with circumradius $p$ that is Pythagorean, i.e., right-angled and with integral sides. After scaling by a factor $k / p$ they obtain a Pythagorean triangle with circumradius $k$. It is easy to show that every Pythagorean triangle has integral area and is thus a Heron triangle. The new implication $(1) \Rightarrow(3)$ of Theorem 1 tells us that among all Heron triangles there are no more integral circumradii than among just the Pythagorean triangles.

The construction above only yields a triangle with relatively prime sides if the integer $k$ is prime. This leaves a rather unsatisfactory feeling, which is resolved by the following theorem. It gives necessary and sufficient conditions for an integer to be the circumdiameter of some Heron triangle with sides that are relatively prime to each other. Theorem 1 will follow as a consequence.

Theorem 2. Let d be a positive integer. Then the following are equivalent:

(1) There is a Pythagorean triangle with circumdiameter $d$ and sides $a, b$, and c satisfying $\operatorname{gcd}(a, b)=\operatorname{gcd}(a, c)=\operatorname{gcd}(b, c)=1$.

(2) There is a Heron triangle with circumdiameter $d$ and sides $a, b$, and $c$ satisfying $\operatorname{gcd}(a, b)=\operatorname{gcd}(a, c)=\operatorname{gcd}(b, c)=1$.

(3) There is a Heron triangle with circumdiameter $d$ and sides $a, b$, and $c$ satisfying $\operatorname{gcd}(a, b, c, d)=1$.

(4) There is a triangle with circumdiameter $d$ and integral sides $a, b$, and $c$ satisfying $\operatorname{gcd}(a, b, c, d)=1$.

(5) We have $d>1$ and every prime divisor $p$ of $d$ satisfies $p \equiv 1 \bmod 4$.

(6) There are positive integers $x$ and $y$ with $x$ even and $\operatorname{gcd}(x, y)=1$, such that $d$ can be written as $d=x^{2}+y^{2}$.

All we will use is some elementary number theory and two formulas. Heron's formula

$$
(4 A)^{2}=(a+b+c)(a+b-c)(a-b+c)(-a+b+c)
$$

relates the area $A$ of a triangle to the lengths $a, b$, and $c$ of its sides. The circumdiameter $d$ is related to these quantities through the equation

$$
2 A d=a b c .
$$

Remark. In Lemma 3 we will deduce from Heron's formula (1) that any triangle with integral sides and rational area is a Heron triangle. Together with (2) this proves the 
implication (4) $\Rightarrow$ (3) of Theorem 2. The fact that (2) implies (1) is a priori not obvious. Neither is the implication (3) $\Rightarrow(2)$, as a triangle with sides $a, b, c$, and circumdiameter $d$ may satisfy $\operatorname{gcd}(a, b, c, d)=1$ even if $a, b$, and $c$ have a divisor in common. The triangle with sides $15,15,24$, and area 108, for example, has circumdiameter 25 . By Theorem 2 the existence of this example does imply that there is a triangle with circumdiameter 25 whose sides are relatively prime. In this case, for $d=25$, the triangle with sides 7, 24, and 25 satisfies all conditions of (1), and therefore also those of (2).

The following lemmas will be useful in proving Theorem 1 and Theorem 2 .

Lemma 3. Any triangle with integral sides and rational area is a Heron triangle.

Proof. The quantity in (1) is both an integer and the square of a rational number. Hence, it is the square of the integer $4 A$. Equation (1) can be rewritten as $(4 A)^{2}=4 b^{2} c^{2}-\left(a^{2}-\right.$ $\left.b^{2}-c^{2}\right)^{2}$. Since -1 is not a square modulo 4 , the quantity $a^{2}-b^{2}-c^{2}$ is even. Therefore, as the parity of any integer, its negative, or its square are all the same, the four factors in the right-hand side of (1) are even as well, and the quantity in (1) is divisible by 16 . It follows that $A^{2}$, and thus $A$, is integral.

Note that it follows from the proof of Lemma 3 that the perimeter of any Heron triangle is even.

For any prime $p$ and any integer $x$, let $v_{p}(x)$ denote the valuation of $x$ at $p$, i.e., the largest integer $m$ for which $p^{m}$ divides $x$. Then $v_{p}$ extends uniquely to a group homomorphism from the group $\mathbb{Q}^{*}$ of nonzero rational numbers to the group $\mathbb{Z}$ of integers.

Lemma 4. Let $p$ be a prime satisfying $p \not \equiv 1 \bmod 4$. Let $s, t$, and $m$ be integers with $m \geq 0$. Then $p^{2 m}$ divides $s^{2}+t^{2}$ if and only if $p^{m}$ divides both $s$ and $t$.

Proof. The "if"-part is obvious. For the "only if"-part, let $n$ be the largest integer for which $p^{n}$ divides both $s$ and $t$. It suffices to show $m \leq n$. By symmetry we may assume $p^{n+1} \nmid s$. Set $s^{\prime}=s p^{-n}$ and $t^{\prime}=t p^{-n}$. Then we have $s^{\prime}, t^{\prime} \in \mathbb{Z}$ and $p \nmid s^{\prime}, \operatorname{sog} \operatorname{gcd}\left(s^{\prime}, p^{2}\right)=1$. From the Euclidean algorithm we find integers $x, y$ such that $x s^{\prime}+y p^{2}=1$, i.e., $x s^{\prime} \equiv$ $1 \bmod p^{2}$. Now from $p^{2 m} \mid s^{2}+t^{2}$ we find $p^{2(m-n)} \mid s^{\prime 2}+t^{\prime 2}$. Suppose $m>n$. Then we conclude $m-n>0$, so $p^{2} \mid s^{\prime 2}+t^{\prime 2}$, and we find $t^{\prime 2} \equiv-s^{\prime 2} \bmod p^{2}$. Therefore we have $\left(x t^{\prime}\right)^{2} \equiv-\left(x s^{\prime}\right)^{2} \equiv-1 \bmod p^{2}$. This contradicts the fact that -1 is neither a square modulo 4 , nor modulo primes $q$ satisfying $q \equiv 3 \bmod 4$. We conclude $m \leq n$, which is what we wanted.

We are now ready to prove our theorems.

Proof of Theorem 2. Reduction modulo 4 shows that at least one of the sides of a Pythagorean triangle other than the hypotenuse is even. Therefore, every Pythagorean triangle has integral area, which proves implication $(1) \Rightarrow(2)$. The implications $(2) \Rightarrow$ $(3) \Rightarrow(4)$ are obvious. For $(4) \Rightarrow(5)$, let $a, b$, and $c$ be the sides of a triangle as in (4). Because the equilateral triangle with sides 1 does not have integral circumdiameter, we get $\max (a, b, c)>1$. The largest chord of a circle being a diameter, we 
find $d \geq \max (a, b, c)>1$. Let $p$ be any prime divisor of $d$ and suppose $p$ satisfies $p \not \equiv 1 \bmod 4$. Without loss of generality, we may assume $v_{p}(a) \geq v_{p}(b) \geq v_{p}(c)$. As $p$ does not divide $\operatorname{gcd}(a, b, c)$, we have $v_{p}(c)=0$. By Lemma 3 the rational area $A=a b c /(2 d)$ is integral. This implies that $p$ divides $a b c$, so $p$ divides $a$. Set $m=v_{p}(2 a)>0$. After rewriting $(1)$ as $(4 A)^{2}+\left(a^{2}+b^{2}-c^{2}\right)^{2}=4 a^{2} b^{2}$, we see that $p^{2 m}$ divides $(4 A)^{2}+\left(a^{2}+b^{2}-c^{2}\right)^{2}$. By Lemma 4 this implies $p^{m} \mid 4 A$ and $p^{m} \mid a^{2}+b^{2}-c^{2}$. Hence, $p$ divides $b^{2}-c^{2}$, and we find $v_{p}(b)=v_{p}(c)=0$. We obtain the contradiction $m=v_{p}(2 a)=v_{p}(2 a b c)=v_{p}(4 A d)>v_{p}(4 A) \geq m$ and conclude that $p$ satisfies $p \equiv 1 \bmod 4$. The implication $(5) \Rightarrow(6)$ is a well-known result, see [3, Theorem 5.15 and its proof]. For $(6) \Rightarrow(1)$, let $x$ and $y$ be as described. Then the triangle with sides $a=2 x y, b=\left|x^{2}-y^{2}\right|$, and $c=x^{2}+y^{2}$ satisfies the requirements of (1).

Corollary 5. Suppose the integer $d$ is the circumdiameter of a triangle with integral sides $a, b$, and $c$. Let $p$ be a prime satisfying $p \neq \equiv 1 \bmod 4$ and set $m=v_{p}(d)$. Then $p^{m}$ divides $\operatorname{gcd}(a, b, c)$.

Proof. After scaling the triangle if necessary, we may assume $\operatorname{gcd}(a, b, c, d)=1$. Then Theorem 2 gives $m=0$.

Proof of Theorem 1. For (1) $\Rightarrow(2)$, let $a, b$, and $c$ be the sides of a Heron triangle with circumradius $k$. Then the circumdiameter $2 k$ is even and by Corollary 5 , so are $a, b$, and $c$. The triangle with sides $a / 2, b / 2$, and $c / 2$ is a Heron triangle by Lemma 3 and has circumdiameter $k$. For $(2) \Rightarrow(3)$, let $a, b$, and $c$ be the sides of a Heron triangle with circumdiameter $k$. Set $r=\operatorname{gcd}(a, b, c, k)$. Then the Heron triangle with sides $a / r$, $b / r$, and $c / r$ has circumdiameter $k / r$ and we have $\operatorname{gcd}(a / r, b / r, c / r, k / r)=1$. From Theorem 2 we find $k / r>1$, so $k / r$ has a prime divisor $p$, which by the same theorem satisfies $p \equiv 1 \bmod 4$. For (3) $\Rightarrow(1)$, let $p$ and $k$ be as in (3). According to Theorem 2 there is a Heron triangle with circumdiameter $p$. Multiplication by $2 k / p$ yields a Heron triangle with circumradius $k$.

\section{References}

[1] Aassila, M.: Some results on Heron triangles. Elem. Math. 56 (2001), 143-146.

[2] Kramer, A.V.; Luca, F.: Some remarks on Heron triangles. Acta Acad. Paedagog. Agriensis Sect. Mat. (N.S.) 27 (2000), 25-38.

[3] Niven, I.; Zuckerman, H.: An introduction to the theory of numbers. Third edition, John Wiley \& Sons, 1972.

Ronald van Luijk

Simon Fraser University

Department of Mathematics

8888 University Drive

Burnaby BC V5A 1S6, Canada

e-mail: ronald_vanluijk@sfu.ca 